\title{
SOME REMARKS ON INEQUALITIES THAT CHARACTERIZE INNER PRODUCT SPACES
}

\author{
JAVIER ALONSO \\ Departamento de Matemáticas \\ Universidad de Extremadura \\ 06071 Badajoz, Spain
}

(Received November 28, 1990 and in revised form March 11, 1991)

ABSTRACT. The purpose of this paper is to obtain some nev characterizations of inner product spaces in the line of the Jordan-von Neumann identity and to make some remarks on similar known characterizations of Day, Delbosco, Rassias and Senechalle, among others.

KEY WORDS AND PHRASES. Inner product spaces, characterizations, inequalities . 1980 AMS SUBJECT CLASSIFICATION CODE. 46B20.

Let $E$ be a real normed linear space. It is well known that $E$ is an inner product space (i.p.s.) if and only if the "parallelogram equality" of P. Jordan and J. von Neumann [1]

$$
\|x+y\|^{2}+\|x-y\|^{2}=2\left(\|x\|^{2}+\|y\|^{2}\right)
$$

holds for every $x, y \in E$. The aim of this paper is to give an account of some characterizations of inner product spaces veaker than the one above in order to make some remarks about them and to obtain some nev characterizations of the same type.

We shall use $S$ to denote the unit sphere of $E$, and $H(x, y)$ the function

$$
H(x, y)=\|x+y\|^{2}+\|x-y\|^{2}-2\left(\|x\|^{2}+\|y\|^{2}\right) \quad(x, y \in E) \text {. }
$$

The first veakening of the Jordan-von Neumann condition vas given by M.M. Day [2] by proving that it can be restricted to unit vectors (i.e. the "rhombus equality"):

$$
E \text { is an i.p.s. } \Leftrightarrow H(x, y)=0 \text { for every } x, y \in S \text {. }
$$

Then I.J. Schoenberg [3] proved that the rhombus equality can be replaced by the "rhombus inequality":

E is an i.p.s. $\Leftrightarrow H(x, y) \sim 0$ for every $x, y \in S$,

where $\sim$ denotes either $\geq$ or $\leq$.

The parallelogram equality can also be changed into the "rectangle inequality" of C. Benítez and M. del Río [4]:

$$
\text { E is an i.p.s. } \Leftrightarrow H(x, y) \sim 0 \text { for every } x, y \in E, x \perp y \text {, }
$$

where $x \perp y$ means "orthogonality" in the G. Birkhoff [5] sense (i.e. $\|x+\lambda y\| \geq\|x\|$ for every $\lambda \in \mathbb{R}$ ).

As D. Amir pointed out [6], implicit in the proof of the above result is that it remains valid if Birkhoff-orthogonality is replaced by any other relation $R$ in $E$ that likerise "admits diagonals" (i.e. such that for every $x, y \in E \backslash\{0\}$ there exists $\alpha>0$ such that $x+\alpha y(x-\alpha y)$ [7]. This happens, for example, if $\perp$ denotes any of the orthogonalities Carlsson [8,9], Diminnie [10,11] and Area [12,13]. (Recall that an 
orthogonality in a normed linear space is a relation that coincides with the usual orthogonality when the norm is induced by an inner product.)

It is known $[5,2,14,15]$ that if $\operatorname{dimE} \geq 3$ the symmetry of Birkhoff-orthogonality is an inner product space characteristic, and that there exist tro-dimensional non inner product spaces (characterized by M.M. Day [2]) vith this property satisfied. Nevertheless, it vas proved also in [4] that if $E$ is a tro-dimensional normed linear space vith symmetric $B$-orthogonality that satisfies a "square inequality" then $E$ is an inner product space. This can be expressed in the folloving way:

$$
\text { E is an i.p.s. } \Leftrightarrow\left[x, y \in S, x \perp^{B} y \Rightarrow y \perp^{B} x \text { and } H(x, y) \leq 0\right] \text {. }
$$

Hovever, there exist non-inner product spaces vith symmetric B-orthogonality and $H(x, y) \geq 0$ for every $x, y \in S, x \perp^{B} y$ (i.e. $\mathbb{R}^{2}$ endozed ith a norm vhose spheres are regular hexagons [4]). It is an open question vhether the square inequality $(\leq)$ alone is characteristic of inner product spaces.

As a particular case of a more general result, D.A. Senechalle [16] proved that if $\epsilon>0$ then

$$
\text { E is an i.p.s. } \Leftrightarrow[x, y \in S,\|x-y\|<\epsilon \Rightarrow H(x, y) \sim 0] \text {. }
$$

Pursuing this line further, and taking into account that the relation " $x \mathrm{H}$ iff $\|x-y\|=\epsilon "$ admits diagonals, we can say that if $\epsilon>0$ then

$$
E \text { is an i.p.s. } \Leftrightarrow[x, y \in E,\|x-y\|=\epsilon \Rightarrow H(x, y) \sim 0] \text {. }
$$

In the next proposition ve shall see that, for particular values of $\epsilon$, these preceding tro characterizations can be improved.

PROPOSITION. Let $D=\left\{2 \cos \frac{k \pi}{2 n}: n=2,3, \ldots, k=1,2, \ldots, n-1\right\} \subset[0,2]$. If $\epsilon \in(0,2) \backslash D$ then

$$
\text { E is an i.p.s. } \Leftrightarrow[x, y \in S,\|x-y\|=\epsilon \Rightarrow H(x, y) \sim 0],
$$

vhere $\sim$ denotes either $\geq$ or $\leq$.

PROOF. It is proved in [17] that if $\epsilon \in(0,2) \backslash D$ then only the inner product spaces satisfy the property

$$
Q_{\epsilon}: \quad x, y \in S,\|x-y\|=\epsilon \Rightarrow H(x, y)=0 .
$$

On the other hand, G. Nordlander [18] proved that if $S_{\mathbb{R}^{2}}$ is the unit sphere of a norm in $\mathbb{R}^{2}$ and $0 \leq \epsilon \leq 2$ then the set $\left\{x+y: x, y \in S_{\mathbb{R}^{2}},\|x-y\|=\epsilon\right\}$ is a simple closed curve that bounds an area $\left(4-\epsilon^{2}\right)$-times the area bounded by $S_{\mathbb{R}^{2}}$. Thus, any normed linear space that satisfies the property

must also satisfy $Q_{\epsilon}$.

$$
x, y \in S,\|x-y\|=\epsilon \Rightarrow H(x, y) \sim 0
$$

Hovever, for $\epsilon \in D$ there exist non-inner product spaces that satisfy $Q_{\epsilon}$ (i.e. if $E$ is the vector space $\mathbb{R}^{2}$ endoved ith a norm vhose spheres are $4 n-g o n s$ then it satisfies $Q_{\epsilon}$ for $\left.\epsilon=2 \cos \frac{k \pi}{2 n}, k=1,2, \ldots, n-1\right)[17]$.

In an extensive paper J. Oman [19] proved that, for tro-dimensional normed linear spaces, one of the vectors in the parallelogram inequality ( $\leq$ ) can be fixed. That is, if $\operatorname{dimE}=2$ then

$E$ is an i.p.s. $\Leftrightarrow$ there exists an $x \in E$ such that $H(x, y) \leq 0$ for every $y \in E$.

Hovever, this result is not true for ( () ). If $E$ is $\mathbb{R}^{2}$ endoped vith the norm $\left\|\left(x_{1}, x_{2}\right)\right\|=\left|x_{1}\right|+\left|x_{2}\right|$ and $x=(1,0)$ then $H(x, y) \geq 0$ for every $y \in E[19]$. 
It is essential for the Oman characterization that $\operatorname{dimE}=2$. If, for example, ve take $E$ to be the vector space $\mathbb{R}^{3}$ endoped with the norm vhose unit ball is the set of points $\left(x_{1}, x_{2}, x_{3}\right)$ defined by the intersection of the tro circular cylinders $x_{1}^{2}+x_{3}^{2} \leq 1$, $x_{2}^{2}+x_{3}^{2} \leq 1$, then any plane containing the origin and the point $x=(0,0,1)$ intersects the unit sphere in an ellipse. Therefore, $H(x, y)=0$ for every $y \in E$. (Similar results for dimensions greater than tro can be found in [19].)

It is an open question whether one vector can also be fixed in the rhombus inequality $(\leq)$.

With regard to norm inequalities of higher degree, Yang Cong-Ren [20] proved that if $p>2$ then

E is an i.p.s. $\Leftrightarrow\|x+y\|^{p}+\|x-y\|^{p} \geq 2\left(\|x\|^{p}+\|y\|^{p}\right)$ for every $x, y \in E$.

(This inequality does not hold for $p<2$. Similar inequalities for this case have been obtained in [20] .)

D. Delbosco [21] proved that if there exists $p>0$ such that $\|x+y\|^{p}+\|x-y\|^{p}=2^{p}$ for every $x, y \in S$, then $E$ must be an inner product space. Therefore, taking into account that if $x, y \in S$ are orthogonal then $\|x \pm y\|=2^{1 / 2}$, it follows that $p=2$. Moreover, taking $\epsilon=2^{1 / 2}$ in the Nordlander sets defined above, it follors that in any tro-dimensional subspace of any normed linear space there alvays exist $x, y \in S$ such that $\|x+y\|=\|x-y\|=2^{1 / 2}$. Therefore, Delbosco's identity holds only for $\mathrm{p}=2$.

Finally, ve shall consider the T.M. Rassias [22] characterization:

E is an i.p.s. $\Leftrightarrow\|x+y\|^{p}+\|x-y\|^{p} \leq 2^{p-1}\left(\|x\|^{p}+\|y\|^{p}\right)$ for every $x, y \in E$ and $p>2$.

Rassias's proof of this result is based on making $p$ tend to 2 in order to obtain the rhombus inequality. Some observations are suggested by this characterization:

Firstly, from the arguments given above, Rassias's inequality does not hold if ve change "క" into " $\geq "$.

Secondly, ve cannot fix $p$. For any $p \geq 2$ the spaces $\ell^{p}$ and $L^{p}$ both satisfy the inequality

$$
\|x+y\|_{p}^{p}+\|x-y\|_{p}^{p} \leq 2^{p-1}\left(\|x\|_{p}^{p}+\|y\|_{p}^{p}\right)
$$

(see e.g. Köthe [23], p.356). Furthermore, ve shall see that the Rassias characterization does not hold if the inequality holds for every $p \geq r$ for some $r>2$. This proves that the hypothesis on $p$ in the Rassias characterization of inner product spaces cannot be reakened.

Taking into account that, for every $c \geq 0$, the functions $f(x)=\left(1+c^{x}\right) 1 / x$ and $g(x)=\left[\frac{1+c^{x}}{2}\right]^{1 / x}, x \geq 0$, are, respectively, monotone decreasing and monotone increasing, it is easy to see that for every $a \geq 0, b \geq 0,0 \leq r \leq p$, the folloving tro inequalities hold:

$$
\begin{aligned}
& \left(a^{p}+b^{p}\right)^{1 / p} \leq\left(a^{r}+b^{r}\right)^{1 / r} \\
& {\left[\frac{a^{p}+b^{p}}{2}\right]^{1 / p} \geq\left[\frac{a^{r}+b^{r}}{2}\right]^{1 / r} .}
\end{aligned}
$$

Bearing this in mind, we have therefore proved that for $p \geq r>2$ and every $x, y$ in $\ell^{r}$ or $L^{r}$, according to the case,

$$
\begin{aligned}
& \left(\|x+y\|_{r}^{p}+\|x-y\|_{r}^{p}\right)^{1 / p} \leq\left(\|x+y\|_{r}^{r}+\|x-y\|_{r}^{r}\right)^{1 / r} \leq \\
& \leq 2^{1-1 / r}\left(\|x\|_{r}^{r}+\|y\|_{r}^{r}\right)^{1 / r} \leq 2^{1-1 / p}\left(\|x\|_{r}^{p}+\|y\|_{r}^{p}\right)^{1 / p} .
\end{aligned}
$$


Hence

$$
\|x+y\|_{r}^{p}+\|x-y\|_{r}^{p} \leq 2^{p-1}\left(\|x\|_{r}^{p}+\|y\|_{r}^{p}\right)
$$

ACKNOWLEDGEMENT. I am grateful to T.M. Rassias for his valuable suggestions .

\section{REFERENCES}

1. JORDAN, P., VON NEUMANN, J. On inner products in linear metric spaces, Ann. of Math. 36 (1935), 719-723.

2. DAY, M.M. Some characterizations of inner product spaces, Trans. Amer. Math. Soc. $\underline{62}(1947), 320-337$.

3. SCHOENBERG, I.J. A remark on M.M. Day's characterization of inner-product spaces and a conjecture of L.M. Blumenthal, Proc. Amer. Math. Soc. 3 (1952), 961-964.

4. BENITEZ, C., DEL RIO, M. Characterization of inner product spaces through rectangle and square inequalities, Rev. Roumaine Math. Pures Appli. 29 (1984), 543-546.

5. BIRKHOFF, G. Orthogonality in linear metric spaces, Duke Math. J. 1 (1935), 169-172.

6. AMIR. D. Characterizations of Inner Product Spaces, Birkhäuser, 1986 .

7. BENITEZ, C. Una propiedad de la ortogonalidad de Birkhoff y una caracterización de espacios prehilbertianos, Collect. Math. 26 (1975), 211-218.

8. CARLSSON, S.0. Orthogonality in normed linear spaces, Ark. Mat. $\underline{4}$ (1962), $297-318$.

9. DEL RIO, M. Ortogonalidad en espacios normados y caracterización de espacios prehilbertianos, Dept. Anal. Matem. Univ. de Santiago de Compostela, Spain, serie $B, 14,1975$.

10. DIMINNIE, C.R. A ned orthogonality relation in normed linear spaces, Math. Nachr. 114 (1983), 197-203.

11. ALONSO, J., BENITEZ, C. Complements on Diminnie orthogonality in normed linear spaces, (preprint).

12. ALONSO, J. Ortogonalidad en Espacios Normados, Ph.D. Thesis, Univ. Extremadura, Badajoz, Spain, 1984.

13. ALONSO, J., BENITEZ, C. Area orthogonality in normed linear spaces, (preprint).

14. JAMES, R.C. Orthogonality and linear functionals in normed linear spaces, Trans. Amer. Math. Soc. 61 (1947), 265-292.

15. JAYES, R.C. Inner products in normed linear spaces, Bull Amer. Math. Soc. $\underline{53}$ (1947), 559-566.

16. SENECHALLE, D.A. Euclidean and non-Euclidean norms in a plane, Illinois Jour. Math. 18 (1971), 281-289.

17. ALONSO, J., BENITEZ, C. Some characteristic and non-characteristic properties of inner product spaces, J. Approx. Theory $\underline{55}$ (1988), 318-325.

18. NORDLANDER, G. The modulus of convexity in normed linear spaces, Arkiv für Matem. $\underline{4}$ (1958), 15-17.

19. OMAN, J. Norm identities vhich characterize inner product spaces, In The Geometry of Metric and Linear Spaces (Ed. A. Dold and B. Eckmann), Springer Lecture Notes no. 490, 116-133, Michigan 1974.

20. YANG CONG-REN Characterization of inner product spaces, Shuxue Jinzhan $\underline{6}$ (1963), 197-200.

21. DELBOSCO, D. Spazi normati con prodotto interno, Rend. Sem. Mat. Univ. Torino 36 $(1977 / 78), 415-419$.

22. RASSIAS, T.M. Ner characterizations of inner product spaces, Bull Sci. Math. (2) 108 (1984), 95-99.

23. KÖTHE, G. Topological Vector Spaces I $\left(2^{\text {nd }}\right.$ ed.), Springer-Verlag, Berlin, Heilderberg, Ner York, 1983. 


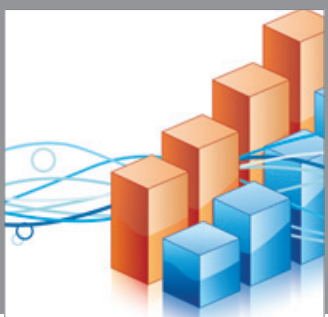

Advances in

Operations Research

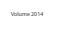

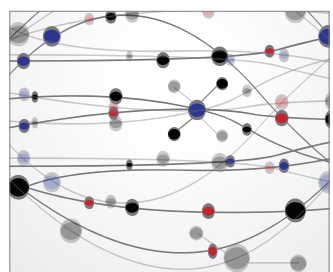

\section{The Scientific} World Journal
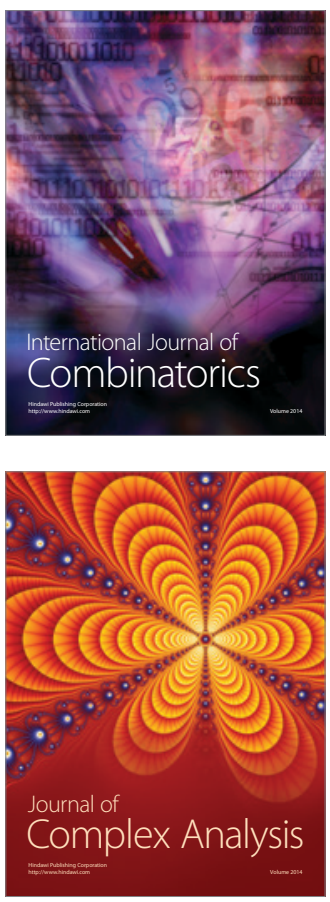

International Journal of

Mathematics and

Mathematical

Sciences
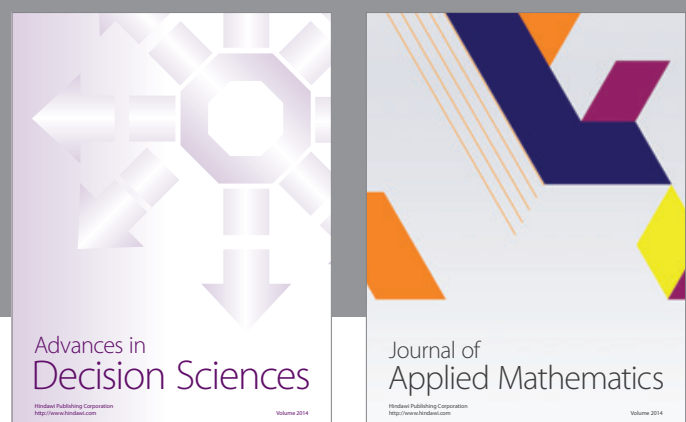

Journal of

Applied Mathematics
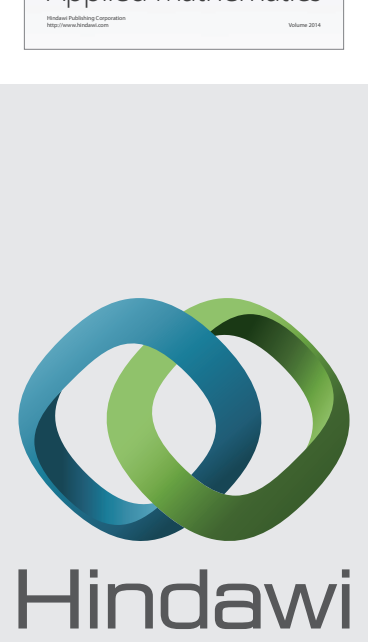

Submit your manuscripts at http://www.hindawi.com
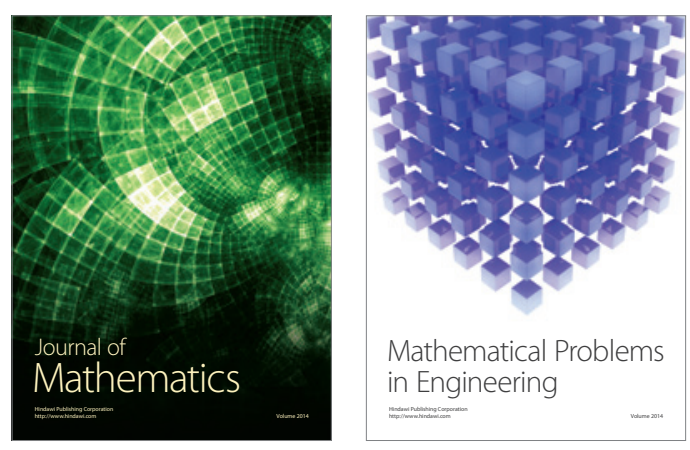

Mathematical Problems in Engineering
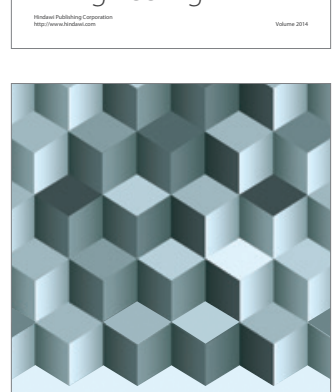

Journal of

Function Spaces
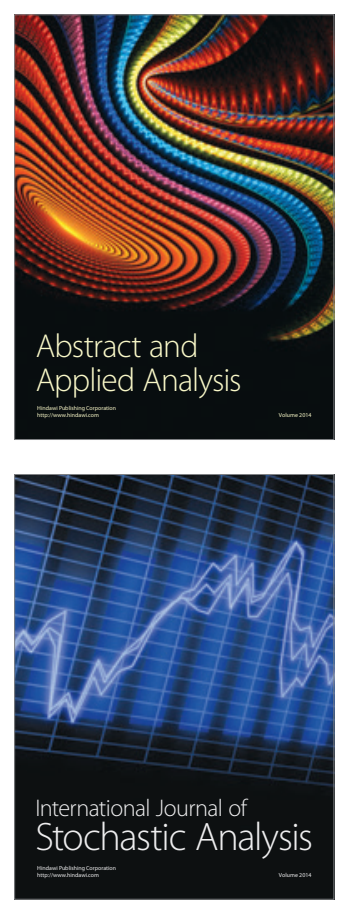

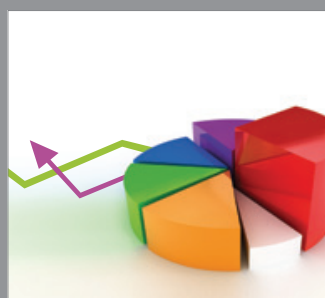

ournal of

Probability and Statistics

Promensencen
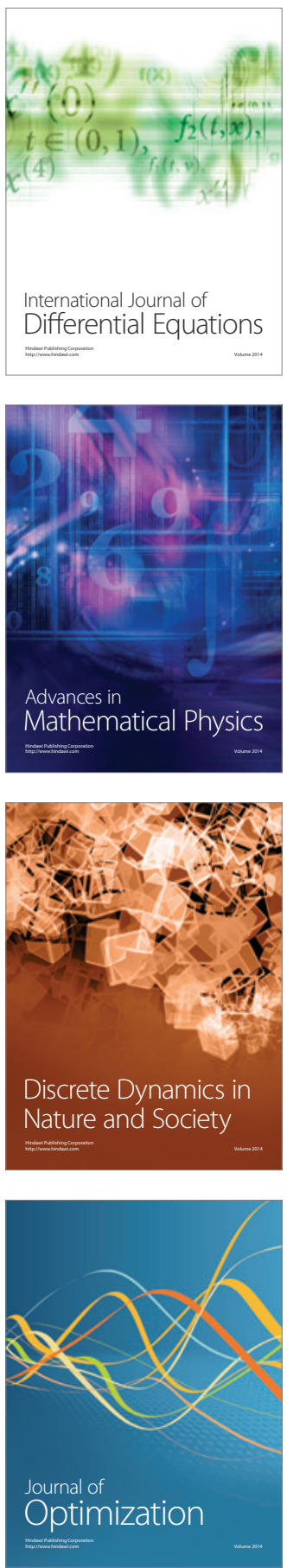